\title{
DNA hypermethylation of sirtuin 1 (SIRT1) caused by betel quid chewing - a possible predictive biomarker for malignant transformation
}

Shajedul Islam,2, Osamu Uehara ${ }^{1,3}$, Hirofumi Matsuoka', Yasuhiro Kuramitsu ${ }^{3}$, Bhoj Raj Adhikari², Daichi Hiraki², Seiko Toraya ${ }^{1}$, Asiri Jayawardena ${ }^{4}$, Ichiro Saito ${ }^{5}$, Malsantha Muthumala ${ }^{6}$, Hiroki Nagayasu', Yoshihiro Abiko ${ }^{2^{*}}$ (D) and Itsuo Chiba'

\begin{abstract}
Background: DNA hypermethylation of tumor suppressor genes is observed in precancerous lesions and oral cancer of individuals with the habits of betel quid (BQ) chewing. SIRT1 has been identified as playing a role in the maintenance of epithelial integrity, and its alteration is often related to carcinogenesis. However, the methylation and transcription status of SIRT1 in patients with BQ chewing-related oral cancer has not been investigated. We examined the methylation status of SIRT1 in paraffin-embedded tissue samples of oral squamous cell carcinoma (OSCC) obtained from BQ chewing and non-chewing patients and in tissue samples from healthy control subjects. In addition, we examined whether the hypermethylation of SIRT1 followed by its transcriptional downregulation in the human gingival epithelial cells could be caused by arecoline, a major component of BQ. Furthermore, we investigated the methylation status of SIRT1 in smear samples of macroscopically healthy buccal mucosa from subjects with a habit of $\mathrm{BQ}$ chewing.

Results: SIRT1 was significantly hypermethylated in tissue samples of OSCC from BQ chewers and non-chewers than in oral mucosa from healthy control subjects. Results also showed that the hypermethylation level of SIRT1 was significantly higher in OSCC of patients with BQ chewing habits than in those of non-chewing habits ( $p<$ 0.05). Our in vitro model showed that hypermethylation is followed by downregulation of the transcriptional level of SIRT1 $(p<0.05)$. The methylation levels of SIRT1 in the smear samples obtained from BQ chewing individuals were significantly higher than those in the samples obtained from individuals that did not chew BQ. The duration of BQ chewing habits was correlated positively to the frequency of SIRT1 hypermethylation $(p<0.05)$.

Conclusions: Our results suggest that DNA hypermethylation of SIRT1 is involved in the occurrence of oral cancer in $\mathrm{BQ}$ chewing patients and that hypermethylation in the oral mucosa of $\mathrm{BQ}$ chewers could be a predictive marker for the occurrence of malignant transformation. This is the first report that showed DNA hypermethylation in clinically healthy oral epithelium of BQ chewers. Our study shows evidence that DNA hypermethylation may be an early event of oral carcinogenesis prior to observable clinical changes.
\end{abstract}

Keywords: Oral cancer, Sirtuin 1, Betel quid chewing, Arecoline, Malignant transformation

\footnotetext{
* Correspondence: yoshi-ab@hoku-iryo-u.ac.jp

${ }^{2}$ Division of Oral Medicine and Pathology, Department of Human Biology

and Pathophysiology, School of Dentistry, Health Sciences University of

Hokkaido, Hokkaido 061-0293, Japan

Full list of author information is available at the end of the article
}

(c) The Author(s). 2020 Open Access This article is distributed under the terms of the Creative Commons Attribution 4.0 International License (http://creativecommons.org/licenses/by/4.0/), which permits unrestricted use, distribution, and reproduction in any medium, provided you give appropriate credit to the original author(s) and the source, provide a link to the Creative Commons license, and indicate if changes were made. The Creative Commons Public Domain Dedication waiver (http://creativecommons.org/publicdomain/zero/1.0/) applies to the data made available in this article, unless otherwise stated. 


\section{Background}

The habit of betel quid (BQ) chewing is considered to be a significant risk factor for oral cancer and oral mucosal diseases in South and Southeast Asian countries [1-3]. BQ may be considered as any quid comprised of betel leaf and a combination of areca nut and slaked lime, with or without tobacco. Each component in BQ may individually, synergistically, and coordinately participate in carcinogenesis [2]. However, the underlying molecular mechanisms for the development of oral cancer remain unclear. Other environmental risk factors, such as tobacco and alcohol, can induce genetic and epigenetic modifications leading to oral cancer [4]. In addition to genetic modifications including mutations [1], DNA hypermethylation of tumor suppressor genes (TSGs) is a common epigenetic event observed in oral cancer [5], and environmental factors have been implicated in the hypermethylation of TSGs [4]. However, few studies have identified DNA hypermethylation in BQ chewing-related oral cancer and precancerous lesions [6-8]. Arecoline, a major alkaloid in areca nuts, is considered to be the most significant procarcinogen present in BQ [9]. Previous studies have demonstrated that arecoline may promote oral cancer by inducing transcriptional downregulation of TSGs $[10,11]$ and that this downregulation may be induced by DNA hypermethylation $[12,13]$. However, the role of arecoline in DNA hypermethylation followed by downregulated transcriptional levels has not been clarified.

Sirtuins (SIRTs) are nicotinamide adenine dinucleotide $\left(\mathrm{NAD}^{+}\right)$-dependent histone deacetylases consisting of seven members (SIRT1-7) [14]. Each of the seven SIRTs has unique characteristics, functions, and localizations [15]. SIRT1 was the first family member to be discovered and is well studied. Deregulated SIRT1 expression was demonstrated previously in various human malignant diseases including oral cancer [16]. However, the physiological relevance of SIRT1 in BQ-related oral cancer remains unexplored. BQ-related oral cancer is often preceded by the development of precancerous lesions, characterized by the disruption of epithelial integrity and, consequently, the transformation to invasive cancer [2]. Intriguingly, SIRT1 has been identified as playing a role in the maintenance of epithelial integrity and contributing to the prevention of both the invasion and metastasis potential of the oral epithelium [17]. From these observations, we hypothesize that decreased SIRT1 expression may occur in oral cancer induced by BQ chewing habit. Since the downregulated expression of SIRT1 has been attributed to DNA hypermethylation [18], we hypothesize that DNA hypermethylation of SIRT1 may be observed followed by its transcriptional downregulated expression in BQ chewing oral cancer patients.

In the present study, we analyzed the methylation status of SIRT1 in paraffin-embedded tissue samples of oral squamous cell carcinoma (OSCC) obtained from BQ chewing and non-chewing patients and in tissues samples from healthy control subjects. In addition, we examined whether the hypermethylation of SIRT1 followed by its transcriptional downregulation in the human gingival epithelial cells could be caused by arecoline, a major component of BQ. Furthermore, we investigated the methylation status of SIRT1 in smear samples of macroscopically healthy buccal mucosa from subjects with a habit of BQ chewing.

\section{Results \\ DNA methylation status of SIRT1 in OSCC obtained from BQ chewing and non-chewing patients}

Seventy-four patients were included in this study (39 males and 35 females). The patients were $24 \mathrm{BQ}$ chewers with OSCC, 25 BQ non-chewers with OSCC, and 25 nonchewing healthy control subjects. Samples of OSCC of BQ chewers were obtained from Sri Lankan patients $(n=24)$, while samples of OSCC of BQ non-chewers $(n=25)$ and samples of healthy controls $(n=25)$ were obtained from Japanese subjects. The demographic data of the participants are listed in Table 1. Pearson's chi-squared test revealed a significant difference in the gender ratio of tissue samples collected from BQ chewers with OSCC from non-chewers with OSCC and healthy control subjects. A significantly greater number of male patients was observed

Table 1 Characteristics of patients and tissue samples

\begin{tabular}{|c|c|c|c|c|c|}
\hline & BQ chewers OSCC & BQ non-chewers OSCC & Healthy controls & Total & $p$ value \\
\hline Samples, N (\%) & $24(32.4)$ & $25(37.8)$ & $25(37.8)$ & $74(100)$ & \\
\hline Gender & & & & & $0.005^{*}$ \\
\hline Male, N (\%) & $19(79.2)$ & $9(36)$ & $11(44)$ & $39(52.7)$ & \\
\hline Female, $N(\%)$ & $5(20.8)$ & $16(64)$ & $14(56)$ & $35(47.3)$ & \\
\hline Age (years) & $56.3 \pm 14.2$ & $61.5 \pm 14.5$ & $54.7 \pm 16.3$ & & $0.253^{* *}$ \\
\hline SIRT1 DNA average methylation level & $45.5 \pm 15.0_{a}$ & $23.7 \pm 14.0_{b}$ & $13.6 \pm 9.8_{c}$ & & $0.000^{* *}$ \\
\hline
\end{tabular}

Data are expressed as mean \pm standard deviation (SD), values in italics represent statistical significance $(p<0.05)$, and subscripts that do not share the same in the row differ at $p<0.05$ in the one-way ANOVA test

$B Q$ betel quid, OSCC oral squamous cell carcinoma

* $p$ value is calculated using Pearson's chi-squared test

${ }^{* *} p$ value is calculated using one-way ANOVA test 
in the BQ chewers with OSCC than in BQ non-chewers with OSCC and healthy controls. A greater number of female subjects comprised the BQ non-chewers with OSCC group than in the BQ chewers OSCC and healthy controls groups. The levels of SIRT1 hypermethylation was significantly altered in the OSCC from BQ chewers and nonchewers groups than in that from healthy controls. The hypermethylation levels were significantly higher in OSCC of BQ chewers compared to non-chewing OSCC patients $(p<0.05$; Table 1$)$.

Furthermore, we examined the promoter methylation status of SIRT1 in OSCC tissue samples obtained from $\mathrm{BQ}$ chewing and non-chewing patients, and oral mucosa samples from healthy control subjects. Multivariable regression analysis was conducted with Bonferroni adjusted $p$ values using DNA methylation as a dependent variable, and age, sex, and groups (analysis I, BQ chewers vs healthy controls; analysis II, BQ non-chewers vs healthy controls; analysis III, BQ chewers vs BQ nonchewers) as independent variables. Analysis I investigated $\mathrm{BQ}$ chewing associated with OSCC, while analysis II investigated BQ non-chewing with OSCC. Analysis III investigated $\mathrm{BQ}$ chewing and non-chewing associated with OSCC. The results showed that SIRT1 was significantly hypermethylated in tissue samples of OSCC from $\mathrm{BQ}$ chewers and non-chewers compared to oral mucosa from healthy control subjects. Results also showed that hypermethylation of SIRT1 was significantly higher in OSCC from $\mathrm{BQ}$ chewing patients than in that from nonchewing patients $(p<0.05$; Table 2$)$.

The effects of arecoline on SIRT1 DNA methylation, mRNA expression, and protein production

In order to confirm whether the hypermethylation of SIRT1 caused by BQ chewing downregulates the transcriptional level of SIRT1 in human gingival epithelial progenitors (HGEPs), HGEPs were stimulated with arecoline, a major component of $\mathrm{BQ}$. The methylation level of SIRT1 in cells treated with arecoline was significantly increased compared to that of control cells $(p<0.05$; Fig. 1a). The expression levels of SIRT1 mRNA in the cells treated with arecoline were significantly decreased compared to the control group $(p<0.05$; Fig. 1b). To determine whether the mRNA expression levels of SIRT1 relates to its protein production, further analysis was performed using western blotting to investigate the effects of arecoline on SIRT1 protein production. The protein levels of SIRT1 (bands of $120 \mathrm{kDa}$ ) were reduced by arecoline treatment in HGEP cells compared to that in controls. On the other hand, the protein levels of GAPDH (bands of $37 \mathrm{kDa}$ ) were the same in all cells (Fig. 1c). The ratio of intensities of SIRT1 to GAPDH (SIRT1/ GAPDH) in controls was considered to be $100 \%$. The ratio of intensities of SIRT1/GAPDH in control and arecoline HGEP cells were $100 \pm 16.2 \%$ and $40.1 \pm 3.3 \%$, respectively. SIRT1 protein levels were reduced by arecoline in HGEP cells $(p<0.05$; Fig. $1 \mathrm{~d})$. These results indicate that SIRT1 mRNA transcription is suppressed by arecoline, resulting in decreased protein production in HGEPs. Together, these results demonstrate that DNA hypermethylation is involved in SIRT1 transcriptional downregulation in HGEP cells following chronic stimulation with arecoline.

\section{Subject characteristics and profiling of SIRT1 DNA methylation}

A total of 70 adult participants were enrolled in the current study, including 14 males and 56 females. Based on the history of oral habits, the participants were classified into two groups: controls (45; healthy, non-chewers) and BQ chewers (25; healthy, chewers). The mean age of controls and BQ chewers was $35.5 \pm 13.6$ years and $39.0 \pm 11.3$ years,

Table 2: Multivariable regression analysis of SIRT1 DNA methylation level (total tissue samples)

\begin{tabular}{|c|c|c|c|c|c|}
\hline Variables & $B$ & Standard error & Beta & $t$ & $p$ value \\
\hline \multicolumn{6}{|l|}{ Analysis I } \\
\hline Age & 0.102 & 0.123 & 0.076 & 0.833 & 1.000 \\
\hline Sex (male 1, female 2) & 0.456 & 4.057 & 0.011 & 0.112 & 1.000 \\
\hline BQ chewers OSCC vs healthy controls & 31.497 & 3.939 & 0.782 & 7.997 & 0.000 \\
\hline \multicolumn{6}{|l|}{ Analysis II } \\
\hline Age & 0.081 & 0.114 & 0.098 & 0.708 & 1.000 \\
\hline Sex & 2.474 & 3.536 & 0.094 & 0.700 & 1.000 \\
\hline BQ non-chewers OSCC vs healthy controls & 5.391 & 1.774 & 0.419 & 3.039 & 0.012 \\
\hline \multicolumn{6}{|l|}{ Analysis III } \\
\hline Age & 0.066 & 0.151 & 0.053 & 0.435 & 1.000 \\
\hline Sex & 2.619 & 4.783 & 0.072 & 0.548 & 1.000 \\
\hline $\mathrm{BQ}$ chewers OSCC vs BQ non-chewers OSCC & 21.005 & 4.806 & 0.587 & 4.370 & 0.000 \\
\hline
\end{tabular}

Values in italics represent statistical significance $(p<0.05) ; p$ value is calculated using Bonferroni adjustment

$B$ unstandardized coefficients, Beta standardized coefficients, BQ betel quid, OSCC oral squamous cell carcinoma 

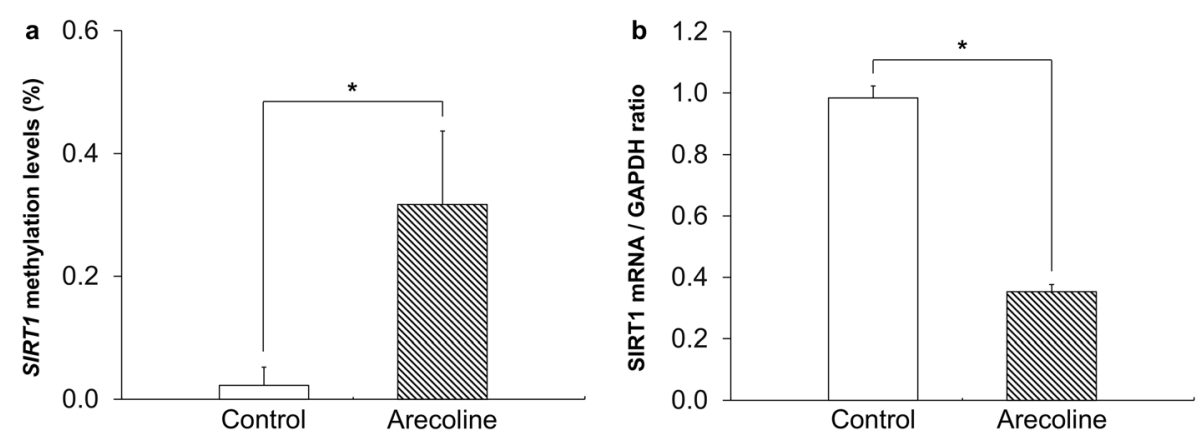

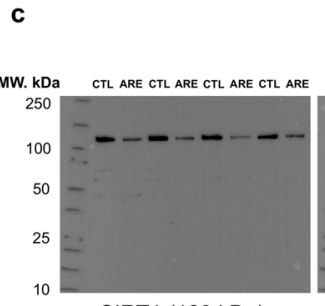

SIRT1 (120 kDa)

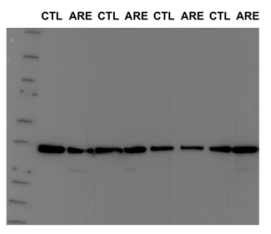

GAPDH (37kDa)

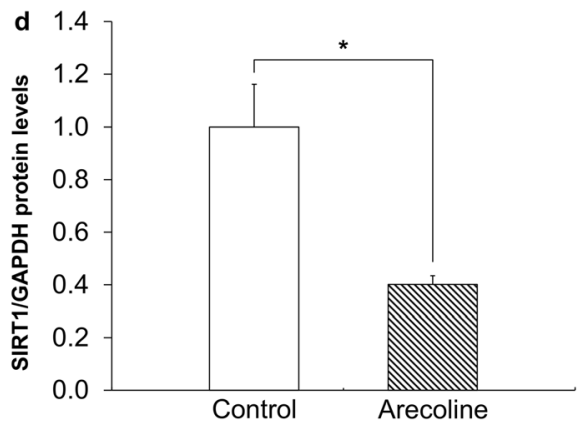

Fig. 1 a The levels of SIRT1 DNA methylation in cells treated with $50 \mu \mathrm{g} / \mathrm{mL}$ arecoline were significantly increased compared to that in control cells. $\mathbf{b}$ The expression levels of SIRT1 mRNA in cells treated with arecoline at $50 \mu \mathrm{g} / \mathrm{mL}$ was significantly decreased compared to that in control cells. c The production of SIRT1 protein (bands of $120 \mathrm{kDa}$ ) was reduced by arecoline treatment compared to controls. As a control, the levels of GAPDH (bands of $37 \mathrm{kDa}$ ) were similar in all cells. $\mathbf{d}$ The ratio of intensities of SIRT1 to GAPDH in control cells was considered to be $100 \%$. The ratio of intensities of SIRT1/GAPDH in control cells and arecoline-treated cells was $100 \pm 16.2 \%$ and $40.1 \pm 3.3 \%$, respectively. The SIRT1 protein levels were reduced by arecoline treatment. Each experiment was performed in triplicate and results with $p$ values of $<0.05$ were considered to be statistically significant. CTL, control; ARE, arecoline

respectively. There were no significant differences observed in terms of gender and age to buccal smear samples of $\mathrm{BQ}$ chewers and non-chewers. The mean chewing years in the BQ chewers group were $7.3 \pm 10.3$ years. Detailed demographics of controls and BQ chewer groups are summarized in Table 3. Of the 25 BQ chewers, 5 (24\%) has used BQ for 6 months or more, 14 (56\%) chewed for $1-10$ years, and 5
(20\%) has used BQ for more than 10 years. The SIRT1 DNA methylation levels in the buccal smear samples of $\mathrm{BQ}$ chewers were significantly higher than in that of nonchewers subjects $(p<0.05$; Table 3$)$.

In this study, we investigated possible correlations between BQ chewing habits and the DNA methylation status of the SIRT1 promoter region in oral mucosal epithelium.

Table 3 Characteristics of human participants and buccal smear samples

\begin{tabular}{|c|c|c|c|c|}
\hline & Control (non-chewer) & Betel quid chewer & Total & $p$ value \\
\hline Samples, N (\%) & $45(64.3)$ & $25(35.7)$ & $70(100)$ & \\
\hline Gender & & & & $0.628^{*}$ \\
\hline Male, $N(\%)$ & $9(20)$ & $5(20)$ & $14(20)$ & \\
\hline Female, $N(\%)$ & $36(80)$ & $20(80)$ & $56(80)$ & \\
\hline Age (years) & $35.5 \pm 13.6$ & $39.5 \pm 12.0$ & & $0.200^{* *}$ \\
\hline \multicolumn{5}{|l|}{ Duration of betel quid chewing habits } \\
\hline$\geq 6$ months, $N(\%)$ & - & $6(24)$ & $25(35.7)$ & \\
\hline $1-10$ years, $N(\%)$ & - & $14(56)$ & & \\
\hline$>10$ years, $N(\%)$ & - & $5(20)$ & & \\
\hline Chewing years (mean $\pm \mathrm{SD}$ ) & - & $7.3 \pm 10.3$ & & \\
\hline SIRT1 DNA average methylation level & $4.0 \pm 4.6$ & $16.5 \pm 23.7$ & & $0.001^{* *}$ \\
\hline
\end{tabular}

Data are expressed as mean \pm standard deviation (SD); values in italics represent statistical significance $(p<0.05)$

${ }^{*} p$ value is calculated using Pearson's chi-square test

${ }^{* *} p$ value is calculated using one-way ANOVA test 
Multivariable regression analysis was conducted with Bonferroni adjusted $p$ values using DNA methylation as a dependent variable, and age, sex, and BQ chewing habit as independent variables. The results showed that $\mathrm{BQ}$ chewing habit was the only significant predictor of DNA methylation $(p<0.05$; Table 4 (A)). Further, among the BQ chewers group, multivariable regression analysis was conducted with Bonferroni adjusted $p$ values using DNA methylation as a dependent variable, and age, sex, and chewing years as independent variables. It was revealed that the duration of chewing habit was significantly correlated to the levels of DNA methylation $(p<0.05$; Table 4 (B)). These results demonstrate that $\mathrm{BQ}$ chewing and the duration of BQ chewing habits are positively correlated with DNA methylation frequency of the SIRT1 gene of oral epithelia.

\section{Discussion}

Results of the present study demonstrated that SIRT1 in OSCC is hypermethylated and that the methylation levels were significantly higher in the OSCC of BQ chewers than in that of non-chewers. Our in vitro model showed that the hypermethylation is followed by downregulation of the transcriptional level of SIRT1. A higher level of methylation of SIRT1 was observed in smear samples obtained from macroscopically healthy buccal mucosa in $B Q$ chewers than in non-chewers. These results suggest that SIRT1 is involved in the oral cancer caused by BQ chewing and that hypermethylation of SIRT1 in the oral mucosa of $\mathrm{BQ}$ chewers may be a predictive marker for detecting early events in multistage carcinogenesis.

Although hypermethylation of SIRT1 has been reported in several cancer tissues [19-21], this is the first demonstration of hypermethylation of SIRT1 in OSCC. We confirmed the occurrence of SIRT1 hypermethylation in OSCC of BQ chewers and non-chewers. We also found that the hypermethylation level of SIRT1 was significantly higher in OSCC of patients with BQ chewing habits than in those of non-chewing habits. These results indicate that the DNA hypermethylation of SIRT1 caused by BQ chewing is involved in BQ-related OSCC. It was not known whether the hypermethylation of SIRT1 caused by BQ chewing is linked to SIRT1 transcription. The extraction of RNA from paraffin-embedded tissue samples remains extremely challenging, and no consensus or standardized isolation method has been described [22]. Therefore, we employed an in vitro model of a daily BQ chewing habit that we showed previously to contain hypermethylated genes [23]. The cells were stimulated with arecoline, a major component of $\mathrm{BQ}$, for a prolonged period according to our previous protocol [24]. We confirmed that significantly high level of methylation of SIRT1 was observed followed by downregulated expression of SIRT1 transcription and protein expression. This hypermethylation of SIRT1 may cause the downregulated expression of SIRT1 observed in OSCC. These results support previous findings suggesting SIRT1 as a tumor suppressor $[17,25]$. SIRT1 has been reported to play a role in maintaining epithelial integrity by inducing the expression of epithelial-cadherin. Downregulation of SIRT1 expression may weaken epithelial-epithelial interaction leading to malignant transformation of the epithelia [17]. The hypermethylation of SIRT1 caused by arecoline in BQ chewers epithelium may be related to the instability of epithelialepithelial interactions causing malignant transformation. It is still unknown how arecoline causes the hypermethylation of SIRT1. The promoter region of SIRT1 possesses a potential regulator of epigenetic factors, methyl-CpG-binding protein 2 (MeCP2) [26]. MeCP2 has been shown to interact with DNA methyltransferase 1 (DNMT1) and recruits the latter to induce SIRT1 promoter methylation [26]. Arecoline was previously documented to promote oral submucosal fibrosis and the progression to oral cancer through pathways involved in transforming growth factor-beta (TGF- $\beta$ ) production $[2,27]$. TGF- $\beta$ is likely to silence SIRT1 epigenetically by inducing the MeCP2 expression, although other possible mechanisms cannot be ruled out [26]. These findings may provide an underlying molecular mechanism of the effect of arecoline on DNA hypermethylation. From these data, we hypothesized that the methylation level of SIRT1 in healthy oral epithelium of BQ chewing subjects is

Table 4 Multivariable regression analysis of SIRT1 DNA methylation level (buccal smear samples)

\begin{tabular}{|c|c|c|c|c|c|}
\hline Variables & $B$ & Standard error & Beta & $t$ & $p$ value \\
\hline \multicolumn{6}{|l|}{ A. Total smear samples $(N=70)$} \\
\hline Age & 0.219 & 0.134 & 0.183 & 1.630 & 0.324 \\
\hline Sex (male 1, female 2) & 3.107 & 4.314 & 0.080 & 0.720 & 1.000 \\
\hline Betel quid chewers vs non-chewers & 11.633 & 3.645 & 0.358 & 3.192 & 0.007 \\
\hline \multicolumn{6}{|l|}{ B. Betel quid chewer group $(N=25)$} \\
\hline Age & 0.189 & 0.396 & 0.095 & 0.478 & 1.000 \\
\hline Sex & 9.529 & 9.782 & 0.164 & 0.974 & 1.000 \\
\hline Chewing years & 1.280 & 0.455 & 0.558 & 2.814 & 0.031 \\
\hline
\end{tabular}

Values in italics represent statistical significance $(p<0.05)$; $p$ value is calculated using Bonferroni adjustment

$B$ unstandardized coefficients, Beta standardized coefficients 
higher than that of non-chewing subjects. We showed that the methylation level of SIRT1 in smear samples obtained from macroscopically healthy buccal mucosa of $\mathrm{BQ}$ chewers is significantly higher than that in the samples of BQ non-chewers. The duration of chewing habits was correlated positively to the frequency of SIRT1 hypermethylation in our data. This observation may support the previous paper that showed increasing the years of quid chewing habits was positively associated with oral cancer [28], wherein SIRT1 hypermethylation may play an important role in the process of their development. Together, these findings indicate that DNA hypermethylation of SIRT1 in epithelium of BQ chewers may be an early event involved in oral carcinogenesis. Previous reports confirmed DNA hypermethylation in precancerous lesions and oral cancer with the habits of $B Q$ chewing $[6,7,28]$. However, no studies have shown alteration of DNA methylation in the macroscopically healthy epithelium of BQ chewers. To be the best of our knowledge, this is the first report that showed DNA hypermethylation in clinically healthy oral epithelium of BQ chewers. This result indicates that DNA hypermethylation may be caused by some carcinogen as an early event of carcinogenesis before their clinical changes. Therefore, examination of SIRT1 hypermethylation, as well as other TSGs in smears of buccal mucosa, could be useful for the detection of early changes caused by BQ chewing habits.

Sampling buccal mucosa and saliva are the two most common non-invasive methods for genetic, epigenetic, and proteomic studies [29]. However, salivary ribonucleases rapidly degrade epithelial cell RNA during collection, and usable RNA has not been extracted from scrapings of buccal mucosa [30]. Therefore, DNA methylation analysis using buccal smear samples may be used as a molecular screen for oral cancer, particularly in areas with limited resources. A few previous studies investigated interactions between BQ chewing habits and DNA methylation using smear samples from buccal OSCC $[8,31]$. However, our study is the first report that shows increased levels of DNA methylation in healthy buccal mucosa samples obtained from BQ chewers. Cigarette smoking and alcohol consumptions are other risk factors for oral cancer [32]. Those habits also cause alteration of DNA methylation [33, 34]. In fact, previous studies demonstrate clear evidence that development of oral cancer follows the same biological pathways irrespective of the source of carcinogenic exposure [35, 36]. Therefore, the hypermethylation of SIRT1 may be a target for the prediction of oral carcinogenesis caused by those habits, as well as BQ chewing. Further investigations are needed to examine this hypothesis.

\section{Conclusions}

In conclusion, our data demonstrate that DNA hypermethylation of SIRT1 occurs in OSCC and normal oral mucosa obtained from $\mathrm{BQ}$ chewers and that the methylation status in buccal smear samples might be considered as an applicable routine oral screening procedure in high-risk populations, particularly in relation to BQ-induced oral cancers. Further studies are necessary to confirm our findings, which might lead to a better understanding of the molecular basis of oral carcinogenesis induced by various environmental exposures.

\section{Materials and methods}

\section{Ethics statement}

All participants in the study provided written informed consent, and the study was approved by the Institutional Review Boards of the Ethics Committee on Human Genetic Research at the Health Sciences University of Hokkaido, Japan (number \# 2016-025), and the Ethical Committee at the University of Peradeniya, Sri Lanka (number \# 7/2004).

\section{Tumor specimen and tissue collection}

Oral squamous cell carcinoma (OSCC) and normal oral mucosa tissue samples were obtained from patients treated surgically. Twenty-four OSCC tissue samples were obtained from patients with $\mathrm{BQ}$ chewing habit in Sri Lanka. Twenty-five OSCC tissue samples were obtained from Japanese patients without BQ chewing habit, and 25 normal oral mucosae were obtained from individuals who underwent oral surgical intervention from 2008 to 2014 at the Health Sciences University of Hokkaido (HSUH) Hospital. The postsurgical tissue sections were formalin-fixed, processed, and paraffin-embedded following standard protocols. None of these patients received chemotherapy or radiotherapy prior to tumor resection. Data on patient demographics were retrieved from the archives of the Oral Medicine and Pathology Department at HSUH, Japan.

\section{Quantitative methylation-specific PCR}

Genomic DNA was extracted from the tissue samples using DNeasy B Blood \& Tissue Kit (Qiagen, Venlo, Netherlands), following the manufacturer's instructions. The extracted DNA samples were treated with sodium bisulfite using the EpiTect ${ }^{\circ}$ Plus Bisulfite Kits (Qiagen). DNA methylation of the SIRT1 gene was analyzed using SYBR green-based quantitative methylation-specific PCR (qMSP). Two sets of primers were used: one for methylated and one for unmethylated DNA sequences [37]. The primers used for SIRT1 gene were as follows (methylated, forward: GGCGAATTTGGTTGTATTATACG, reverse: GAACGAAAACTATTACGTCTACCG; unmethylated, forward: GGGGTGAATTTGGTTGTATTATATG, reverse: AAACAAAAACTATTACATCTACCACT). For PCR, the bisulfite-treated DNA template was mixed with KAPA SYBR FAST qPCR Kit and a pair of primers. The 
PCR conditions included initial incubation at $50^{\circ} \mathrm{C}$ for 2 min, denaturing at $95^{\circ} \mathrm{C}$ for $10 \mathrm{~min}, 50$ cycles of denaturing at $95^{\circ} \mathrm{C}$ for $15 \mathrm{~s}$, and annealing at $58^{\circ} \mathrm{C}$ for $1 \mathrm{~min}$. After PCR amplification, a dissociation curve was generated to confirm the size of the PCR product. The percentage of DNA methylation in a sample was estimated using the following formula:

$$
\text { Methylated DNA } \quad(\%)=\frac{M}{M+U} \times 100=\frac{1}{1+\frac{U}{M}} \times 100
$$

$=\frac{1}{1+2^{(-\Delta C t)}} \times 100$,

where $M$ is the copy number of methylated DNA, $U$ is the copy number of unmethylated DNA, and $\Delta \mathrm{Ct}=$ $\mathrm{Ct}_{U}-\mathrm{Ct}_{M}$ [38]. Each experiment was performed in triplicate. Data are expressed as mean \pm standard deviation (SD).

\section{Cell culture and arecoline exposure}

Human gingival epithelial progenitors (HGEPs) and primary keratinocytes derived from healthy gingival epithelium were purchased from CELLnTEC Advanced Cell Systems (Basel, Switzerland) and cultured in CnT-Prime epithelial culture medium (CELLnTEC Advanced Cell Systems) at $37{ }^{\circ} \mathrm{C}$ in a humidified atmosphere of $95 \%$ air and $5 \% \mathrm{CO}_{2}$. HGEPs were spread onto $100 \mathrm{~mm}$ tissue culture plates at a density of $4.0 \times 10^{4}$ cells $/ \mathrm{mL}$. Arecoline (arecoline hydrobromide) was purchased from Sigma-Aldrich (St. Louis, MO). Following overnight incubation, the HGEPs were treated with arecoline at a concentration of $50 \mu \mathrm{g} / \mathrm{mL}$. The concentration of arecoline used in this study was as described in previous experiments [24]. Briefly, arecoline at the concentration of $50 \mu \mathrm{g} / \mathrm{mL}$ had no cytotoxic effect on the cells stimulated, even for a prolonged period, the method of alternating between 3 days with $50 \mu \mathrm{g} / \mathrm{mL}$ of arecoline and 3 days without arecoline for 1 month was selected. The untreated samples were used as controls (Fig. 2).

\section{Quantitative methylation-specific PCR}

Genomic DNA was extracted from the HGEPs using DNeasy ${ }^{\circ}$ Blood \& Tissue Kit (Qiagen), following the manufacturer's instructions. The extracted DNA samples were treated with sodium bisulfite using the EpiTect ${ }^{\circ}$ Plus Bisulfite Kits (Qiagen), and DNA methylation of the SIRT1 gene was performed using the method described previously [38]. Each experiment was performed in triplicate. Data are expressed as mean \pm SE of the DNA methylation.

\section{Real-time quantitative reverse-transcription PCR}

Total RNA was extracted from the HGEPs using the RNeasy ${ }^{\circ}$ Mini Kit (Qiagen) following the manufacturer's instructions. Total RNA was reverse transcribed into cDNA using a ReverTra Ace ${ }^{\bullet}$ qPCR RT Master Mix (Toyobo, Osaka, Japan). The cDNA levels were measured using the LightCycler ${ }^{\circ}$ Nano System (Roche Diagnostics, Basel, Switzerland). Two sets of primer were used (SIRT1, forward: GCGATTGGGTACCGAGATAA, reverse: TTGCATGTGAGGCTCTATCC; GAPDH, forward: GTGAAGGTCGGAGTCAAC, reverse: GTTGAG GTCAATGAAGGG) [39, 40]. For qRT-PCR, cDNA was mixed with KAPA SYBR FAST qPCR Kit (Nippon Genetics, Tokyo, Japan) and a pair of primers. The PCR conditions included initial incubation at $50^{\circ} \mathrm{C}$ for $2 \mathrm{~min}$, denaturing at $95^{\circ} \mathrm{C}$ for $10 \mathrm{~min}, 40$ cycles of denaturing at $95^{\circ} \mathrm{C}$ for $15 \mathrm{~s}$, and annealing at $60^{\circ} \mathrm{C}$ for $1 \mathrm{~min}$. The relative expression of each mRNA was calculated as the $\mathrm{Ct}$ (the value obtained by subtracting the $\mathrm{Ct}$ value of the GAPDH mRNA from the Ct value of the target mRNA) using the $\Delta \Delta \mathrm{Cq}$ method [41]. Specifically, the amount of target mRNA relative to GAPDH mRNA is expressed as $2^{-(\Delta \mathrm{Ct})}$. Each experiment was performed in triplicate. Data are expressed as mean \pm standard error (SE) of the ratio of the target mRNA to GAPDH mRNA.

\section{Western blotting analysis}

Proteins were extracted from the HGEPs using lysis buffer [50 mM Tris HCL, pH 7.5; 10 mM EDTA, pH 7.5; 165 $\mathrm{mM} \mathrm{NaCl} ; 10 \mathrm{mM} \mathrm{NaF} ; 1 \%$ Nonidet P-40; 1 mM PMSF; 1 $\mathrm{mM} \mathrm{NaVO} ; 10 \mu \mathrm{g} / \mathrm{mL}$ leupeptin; and $10 \mu \mathrm{g} / \mathrm{mL}$ aprotinin]. The lysis reaction was carried out for $1 \mathrm{~h}$ at $4{ }^{\circ} \mathrm{C}$. The samples were centrifuged at $15,000 \mathrm{rpm}$ for $30 \mathrm{~min}$ at $4{ }^{\circ} \mathrm{C}$, and the supernatant was used as sample. Protein concentration was quantified by Lowry's protein assay. Fifteen micrograms of protein samples were used for western blotting analysis. Sodium dodecyl sulfate-polyacrylamide gel electrophoresis (SDS-PAGE) was carried out in precast gels (4-20\% gradient of polyacrylamide; MiniPROTEAN TGX Gels; Bio-Rad, Hercules, CA, USA).

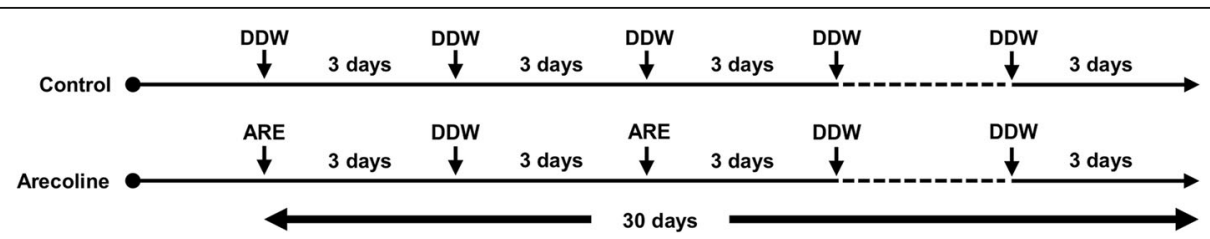

Fig. 2 Flow chart of cell culture. Human gingival epithelium progenitors (HGEPs), cells were treated with arecoline at a concentration of $50 \mathrm{\mu g} /$ $\mathrm{mL}$. The culture media was replaced every 3 days, alternating media with and without arecoline for 30 days. Untreated samples were used as controls. DDW, double-distilled water; ARE, arecoline 
After electrophoresis, gels were transferred electrophoretically onto polyvinylidene difluoride membranes (Immobilon-P; Millipore, Bedford, MA, USA) and blocked for $1 \mathrm{~h}$ with Tris-buffered saline (TBS) containing 5\% skimmed milk. Blocked membranes were washed twice with TBS containing $0.05 \%$ Tween- 20 solution.

The following primary antibodies were used: mouse monoclonal antibody against SIRT1 (dilution 1:1000, \#ab110304; Abcam, Cambridge, MA, USA) and rabbit monoclonal antibody against GAPDH (dilution 1:5000, \#CST5174; Cell Signaling Technology, Danvers, Massachusetts, USA). Membranes were incubated with the primary antibody overnight at $4{ }^{\circ} \mathrm{C}$, washed three times with TBS containing $0.05 \%$ Tween-20 solution, and incubated with a horseradish peroxidase-conjugated secondary antibody (dilution 1:10,000; Jackson ImmunoResearch Laboratories Inc., West Grove, PA, USA) for 1 $\mathrm{h}$ at room temperature. Bands of SIRT1 and GAPDH were visualized by the enhanced chemiluminescence system (Clarity Max ${ }^{\text {TM }}$ Western ECL Substrate; Bio-Rad) and LuminoGraph I (ATTO Corporation, Tokyo, Japan) and recorded using ImageSaver6 software (ATTO Corporation). Expression levels of SIRT1 and GAPDH in cells treated with or without arecoline were quantified by analyzing the intensity of each band using CS Analyzer4 software (ATTO Corporation). Each experiment was performed in triplicate. Data are expressed as mean $\pm \mathrm{SE}$ of the ratio of the target protein to GAPDH protein.

\section{Buccal smear samples and clinicopathological data collection}

Prior to sample collection, the nature of the study was fully explained to all participants. Information obtained from the interview included socioeconomic and demographic characteristics; personal and family histories; risk factors for oral cancer, such as lifestyle, alcohol drinking, tobacco smoking, and $\mathrm{BQ}$ chewing including its frequency; and the added use of betel leaf, areca nut, slaked lime, and tobacco. Clinic attendees of at least 20 years of age and with the ability to complete the questionnaires by an interview and clinical oral examinations were eligible to participate. The presence of oral mucosal lesions was evaluated and documented by a registered dentist, based on the recommendations of the World Health Organization [42]. Participants having oral mucosal lesions or any systemic disorders (such as diabetes, immune-compromised, or genetic diseases) were excluded from this study. A convenience sample of 70 study subjects was recruited and classified into two groups: controls (45 healthy, non-chewers) and BQ chewers (25 healthy, chewers). Both the controls and BQ chewer groups were non-smokers and non-drinkers. Subjects who had chewed one BQ per day for at least 6 months were considered as chewers.
For oral cancer screenings, samples for biomarker testing should be easily available. Thus, the present study used samples obtained by buccal smear, which is noninvasive and easy to perform and which may assist in screening for oral cancers, particularly in areas with limited resources. Buccal smear samples were collected by research staff according to the manufacturer's instructions (Qiagen). Briefly, the participants were restricted in eating and drinking for $30 \mathrm{~min}$ prior to collection, and it was verified that the participant's mouth was empty. The swab sticks were removed from the package carefully to avoid contaminating the tip of the swab with gloves or against any surface. The swab was firmly rubbed and rotated along the inside of the cheek for 5-10 times, and to ensure that the entire tip was in contact with the cheek, this step was repeated on the other cheek. The swab stick was removed from the mouth, being careful not to touch swab tips with any other surface such as teeth, lips, or other surfaces. The swab was placed directly into the tube containing the DNA stabilizing reagent Gentra ${ }^{\circ}$ Puregene ${ }^{\circ}$ buccal cell kits (Qiagen), and the tube was labeled with identifying information. Samples were stored at $-20{ }^{\circ} \mathrm{C}$ until shipment on ice to HSUH for testing.

\section{Quantitative methylation-specific PCR}

Genomic DNA was extracted from the buccal smear samples using Gentra ${ }^{\circ}$ Puregene ${ }^{\circ}$ buccal cell kits (Qiagen), following the manufacturer's instructions. The extracted DNA samples were treated with sodium bisulfite using the EpiTect ${ }^{\bullet}$ Plus Bisulfite Kits (Qiagen), and DNA methylation of the SIRT1 gene was performed using the methods described previously [38]. Each experiment was performed in triplicate. Data are expressed as mean \pm $\mathrm{SD}$ of the DNA methylation.

\section{Statistical analysis}

Statistical analysis was performed on a database using IBM SPSS Statistics 23 (IBM, Armonk, NY). Pearson's chi-squared test was used to analyze gender differences in groups of tissue samples (BQ chewers OSCC, BQ non-chewers OSCC, and healthy controls) and in buccal smear samples (control and BQ chewer). One-way ANOVA test was performed to analyze the age and DNA methylation level differences in tissue samples and buccal smear sample subjects. Multivariable regression analysis was performed with Bonferroni adjusted $p$ values for multiple comparisons in tissue samples of OSCC and healthy control subjects, and buccal smear samples of BQ chewers and non-chewers subjects. Mann-Whitney $U$ test was performed between groups of arecoline-treated and untreated samples. Results with $p$ values of $<0.05$ were considered to be statistically significant. 


\section{Abbreviations}

BQ: Betel quid; DNMT1: DNA methyltransferase 1; MeCP2: Methyl-CpGbinding protein 2; OSCC: Oral squamous cell carcinoma; SIRT1: Sirtuin 1; SIRTs: Sirtuins; TGF- $\beta$ : Transforming growth factor-beta; TSGs: Tumor suppressor genes

\section{Acknowledgements}

The authors thank the research participants for their contributions to their research. The authors also thank Dr. Koki Yoshida, Dr. Tetsuro Morikawa, Dr. Durga Paudel, Dr. Rie Takai, and Dr. Fumiya Harada for their valuable research assistance.

\section{Authors' contributions}

$\mathrm{SI}, \mathrm{OU}, \mathrm{HM}, \mathrm{IC}, \mathrm{YA}$, and YK conceived and designed the study. SI performed the experiments. SI, HM, BRA, and DH performed statistical analyses. SK, AJ, IS, MM, HN, and IC contributed with participant enrolment. SI, YA, YK, and IC wrote the manuscript. All authors read and approved the final manuscript.

\section{Funding}

This research was supported by the Grant-in-Aid for Scientific Research (B) and $(\mathrm{C})$ from the Ministry of Education Science Sports and Culture of Japan (no. 25305036 and 17K11916).

\section{Availability of data and materials}

All data generated or analyzed during this study are included in this published article.

\section{Ethics approval and consent to participate}

This study follows international and national regulations in accordance with the Declaration of Helsinki and was approved by the Ethics Committee of Human Genetic Research at the Health Sciences University of Hokkaido, Japan, and the Ethical Committee at the University of Peradeniya, Sri Lanka. All patients signed informed consent before being included in this study, and their personal data were maintained confidential during this research.

\section{Consent for publication}

Not applicable.

\section{Competing interests}

The authors declare that they have no competing interests.

\section{Author details}

'Division of Disease Control and Molecular Epidemiology, Department of Oral Growth and Development, School of Dentistry, Health Sciences University of Hokkaido, Hokkaido 061-0293, Japan. ²Division of Oral Medicine and Pathology, Department of Human Biology and Pathophysiology, School of Dentistry, Health Sciences University of Hokkaido, Hokkaido 061-0293, Japan. ${ }^{3}$ Research Institute of Cancer Prevention, Health Sciences University of

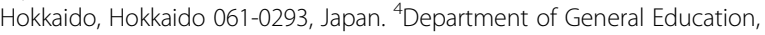
School of Dental Medicine, Tsurumi University, Kanagawa 230-8501, Japan. ${ }^{5}$ Department of Pathology, School of Dental Medicine, Tsurumi University, Kanagawa 230-8501, Japan. ${ }^{6}$ Department of Oral and Maxillofacial Surgery, Army Hospital, Colombo, Sri Lanka. ${ }^{7}$ Division of Oral and Maxillofacial Surgery, Department of Human Biology and Pathophysiology, School of Dentistry, Health Sciences University of Hokkaido, Hokkaido 061-0293, Japan.

Received: 29 September 2019 Accepted: 30 December 2019 Published online: 13 January 2020

\section{References}

1. Chiba I, Muthumala M, Yamazaki Y, Zaman AU, liuka T, Amemiya A, et al. Characteristics of mutations in the p53 gene of oral squamous cell carcinomas associated with betel-quid chewing in Sri Lanka. Int J Cancer. 1998;77:839-42.

2. Islam S, Muthumala M, Matsuoka H, Uehara O, Kuramitsu Y, Chiba I, et al. How each component of betel quid is involved in oral carcinogenesis: mutual interactions and synergistic effects with other carcinogens---a review article. Curr Oncol Rep. 2019;21:53.

3. Lee $\mathrm{CH}$, Ko AM, Warnakulasuriya S, Yin BL, Sunarjo, Zain RB, et al. Intercountry prevalences and practices of betel-quid use in south, southeast and eastern Asia regions and associated oral preneoplastic disorders: an international collaborative study by Asian betel-quid consortium of south and east Asia. Int J Cancer. 2011:129:1741-51.

4. Ghantous Y, Schussel JL, Brait M. Tobacco and alcohol-induced epigenetic changes in oral carcinoma. Curr Opin Oncol. 2018;30:152-8.

5. Irimie Al, Ciocan C, Gulei D, Mehterov N, Atanasov AG, Dudea D. BerindanNeagoe. Current insights into oral cancer epigenetics. Int J Mol Sci. 2018;19:670.

6. Kaur J, Demokan S, Tripathi SC, Macha MA, Begum S, Califano JA, Ralhan R. Promoter hypermethylation in Indian primary oral squamous cell carcinoma. Int J Cancer. 2010;127:2367-73.

7. Takeshima M, Saitoh M, Kusano K, Nagayasu H, Kurashige $Y$, Malsantha M, et al. High frequency of hypermethylation of p14, p15, and p16 in oral precancerous lesions associated with betel-quid chewing in Sri Lanka. J Oral Pathol Med. 2010:37:475-9.

8. Huang YK, Peng BY, Wu CY, Su CT, Wang HC, Lai HC. DNA methylation of PAX1 as a biomarker for oral squamous cell carcinoma. Clin Oral Investig. 2014;18:801-8.

9. Gupta PC, Pindborg JJ, Mehta FS. Comparison of carcinogenicity of betel quid with and without tobacco: an epidemiological review. Ecol Dis. 1982;1:213-9.

10. Ji WT, Yang SR, Chen JY, Cheng YP, Lee YR, Chiang MK, Chen HR. Arecoline downregulates levels of p21 and p27 through the reactive oxygen species/ mTOR complex 1 pathway and may contribute to oral squamous cell carcinoma. Cancer Sci. 2012;103:1221-9.

11. Chuerduangphui J, Ekalaksananan $T$, Chaiyarit $P$, Patarapadungkit $N$ Chotiyano A, Kongyingyoes $B$, et al. Effects of arecoline on proliferation of oral squamous cell carcinoma cells by dysregulating c-Myc and miR-22, directly targeting oncostatin M. PLoS One. 2018;13:1-16.

12. Lai ZL, Tsou YA, Fan SR, Tsai MH, Chen L, Chang NW, et al. Methylationassociated gene silencing of RARB in areca carcinogens induced mouse oral squamous cell carcinoma. Biomed Res Int. 2014;2014:378358.

13. Saikia JR, Schneeweiss FH, Sharan RN. Arecoline-induced changes of polyADP-ribosylation of cellular proteins and its influence on chromatin organization. Cancer Lett. 1999:139:59-65.

14. Sebastian C, Satterstrom FK, Haigis MC, Mostoslavsky R. From sirtuin biology to human diseases: an update. J Biol Chem. 2012;287:42444-52.

15. Carafa V, Rotili D, Forgione M, Cuomo F, Serretiello E, Hailu G, et al. Sirtuin functions and modulation: from chemistry to the clinic. Clin Epigenetics. 2016;8:61.

16. Islam S, Abiko Y, Uehara O, Chiba I. Sirtuin 1 and oral cancer (Review). Oncol Lett. 2019:17:729-38.

17. Chen IC, Chiang WF, Huang HH, Chen PF, Shen YY, Chiang HC. Role of SIRT1 in regulation of epithelial-to-mesenchymal transition in oral squamous cell carcinoma metastasis. Mol Cancer. 2014;13:254.

18. Furuya TK, da Silva PN, Payão SL, Rasmussen LT, de Labio RW, Bertolucci PH, et al. SORL1 and SIRT1 mRNA expression and promoter methylation levels in aging and Alzheimer's disease. Neurochem Int. 2012;61:973-5.

19. Frazzi R, Zanetti E, Pistoni M, Tamagnini I, Valli R, Braglia L, Merli F. Methylation changes of SIRT1, KLF4, DAPK1 and SPG20 in B-lymphocytes derived from follicular and diffuse large B-cell lymphoma. Leuk Res. 2017:57:89-96.

20. Frazzi R, Zanetti E, Pistoni M, Tamagnini I, Valli R, Merli F. Abstract 4451: Different patterns of SIRT1, KLF4, DAPK1 and SPG20 methylation in B lymphocytes correlate with the clinical parameters of non-Hodgkin lymphoma subtypes. Cancer Res. 2016;76:4451. DOI. https://doi.org/10.1158/ 1538-7445.AM2016-4451

21. Lisboa LCF, Gigek C de O, Silva PNO, Leal MF, Lima EM, Calcagno DQ, Assumpção PP de, et al. Protein expression and promoter DNA methylation of SIRT1 and 3 in gastric cancer. 2012. Available from: https://pdfs. semanticscholar.org/9642/0d6cbf5f54157d88127a9cf246d9c5565f96.pdf

22. Bohmann K, Hennig G, Rogel U, Poremba C, Mueller BM, Fritz P, et al. RNA extraction from archival formalin-fixed paraffin-embedded tissue: a comparison of manual, semiautomated, and fully automated purification methods. Clin Chem. 2009:55:1719-27.

23. Takai R, Uehara O, Harada F, Utsunomiya M, Chujo T, Yoshida K, et al. DNA hypermethylation of extracellular matrix-related genes in human periodontal fibroblasts induced by stimulation for a prolonged period with lipopolysaccharide derived from Porphyromonas gingivalis. J Periodontal Res. 2016;51:508-17

24. Uehara O, Takimoto K, Morikawa T, Harada F, Takai R, Adhikari BR, et al. Upregulated expression of MMP-9 in gingival epithelial cells induced by prolonged stimulation with arecoline. Oncol Lett. 2017;14:1186-92.

25. Kang YY, Sun FL, Zhang Y, Wang Z. SIRT1 acts as a potential tumor suppressor in oral squamous cell carcinoma. J Chinese Med Assoc. 2018;81: 416-22. 
26. Volkmann I, Kumarswamy R, Pfaff N, Fiedler J, Dangwal S, Holzmann A, et al MicroRNA-mediated epigenetic silencing of sirtuin 1 contributes to impaired angiogenic responses. Circ Res. 2013;113:997-3.

27. Khan I, Kumar N, Pant I, Narra S, Kondaiah P. Activation of TGF- $\beta$ pathway by areca nut constituents: a possible cause of oral submucous fibrosis. PLoS One. 2012;7:e51806.

28. Shridhar K, Walia GK, Aggarwal A, Gulati S, Geetha AV, Prabhakaran D, et al. DNA methylation markers for oral pre-cancer progression: a critical review. Oral Oncol. 2016;53:1-9.

29. Theda C, Hwang SH, Czajko A, Loke YJ, Leong P, Craig JM. Quantitation of the cellular content of saliva and buccal swab samples. Sci Rep. 2018;8:6944.

30. Ceder O, Van Dijken J, Ericson T, Kollberg H. Ribonuclease in different types of saliva from cystic fibrosis patients. Acta Paediatr Scand. 1985;74:102-6.

31. Yang CC, Wu CH, Chang CF, Hung CP, Sy M, Lin HS, et al. DNA methylation confers clinical potential to predict the oral cancer prognosis. Clin Res Trials. 2018. DOI: 10.15761/CRT.1000232.

32. Scully C. Oral cancer aetiopathogenesis; past, present and future aspects. Med Oral Patol Oral Cir Bucal. 2011;16:e306-11.

33. Wang TH, Hsia SM, Shih YH, Shieh TM. Association of smoking, alcohol use, and betel quid chewing with epigenetic aberrations in cancers. Int J Mol Sci. 2017;18:pii: E1210.

34. Lee KW, Pausova Z. Cigarette smoking and DNA methylation. Front Genet. 2013;4:132.

35. Lunde ML, Warnakulasuriya S, Sand L, Hirsch JM, Vasstrand EN, Ibrahim SO. Gene expression analysis by CDNA microarray in oral cancers from two Western populations. Anticancer Res. 2010:30:1083-91.

36. Dysvik B, Vasstrand EN, Løvlie R, Elgindi OA, Kross KW, Aarstad HJ, et al. Gene expression profiles of head and neck carcinomas from Sudanese and Norwegian patients reveal common biological pathways regardless of race and lifestyle. Clin Cancer Res. 2006;12:1109-20.

37. Gigek OC. SIRT1, IGFBP-3 and CAV1 promoter DNA methylation in aging Transl Med. 2014;4:133.

38. Lu L1, Katsaros D, de la Longrais IA, Sochirca O, Yu H. Hypermethylation of let-7a-3 in epithelial ovarian cancer is associated with low insulin-like growth factor-II expression and favorable prognosis. Cancer Res. 2007;67: 10117-22.

39. Li S, Li C, Ryu HH, Lim SH, Jang WY, Jung S. Bacitracin inhibits the migration of U87-MG glioma cells via interferences of the integrin outside-in signaling pathway. J Korean Neurosurg Soc. 2016:59:106-16.

40. Murray MY, Rushworth SA, Zaitseva L, Bowles KM, MacEwan DJ. Attenuation of dexamethasone-induced cell death in multiple myeloma is mediated by miR-125b expression. Cell Cycle. 2013;12:2144-53.

41. Livak KJ, Schmittgen TD. Analysis of relative gene expression data using realtime quantitative PCR and the 2- $\Delta \Delta C T$ method. Methods. 2001;25:402-8.

42. Kramer I, Pindborg JJ, Bezroukov V, Infirri JS. Guide to epidemiology and diagnosis of oral mucosal diseases and conditions. World Health Organization. Community Dent Oral Epidemiol. 1980;8:1-26.

\section{Publisher's Note}

Springer Nature remains neutral with regard to jurisdictional claims in published maps and institutional affiliations.

Ready to submit your research? Choose BMC and benefit from:

- fast, convenient online submission

- thorough peer review by experienced researchers in your field

- rapid publication on acceptance

- support for research data, including large and complex data types

- gold Open Access which fosters wider collaboration and increased citations

- maximum visibility for your research: over $100 \mathrm{M}$ website views per year

At $\mathrm{BMC}$, research is always in progress.

Learn more biomedcentral.com/submissions 